\title{
Near-Field Spot for Localized Light-Excitation of a Single Fluorescent Molecule
}

\author{
Muhammad Shemyal NISAR ${ }^{1}$, Yujun CUI ${ }^{1}$, Kaitong DANG ${ }^{1}$, \\ Liyong JIANG ${ }^{2}$, and Xiangwei ZHAO ${ }^{*}$
}

\author{
${ }^{1}$ State Key Laboratory of Bioelectronics, School of Biological Science and Medical Engineering, Southeast University, \\ Nanjing 210096, China \\ ${ }^{2}$ Department of Physics, School of Science, Nanjing University of Science and Technology, Nanjing 210094, China \\ "Corresponding author: Xiangwei ZHAO_E-mail: xwzhao@seu.edu.cn
}

\begin{abstract}
Zero-mode waveguides have become important tools for the detection of single molecules. There are still, however, serious challenges because large molecules need to be packed into nano-holes. To circumvent this problem, we investigate and numerically simulate a novel planar sub-wavelength 3-dimension (3D) structure, which is named as near-field spot. It enables the detection of a single molecule in highly concentrated solutions. The near-field spot can produce evanescent waves at the dielectric/water interface, which exponentially decay as they travel away from the dielectric/water interface. These evanescent waves are keys for the detection of fluorescently tagged single molecules. A numerical simulation of the proposed device shows that the performance is comparable with a zero-mode waveguide. Additional degrees-of-freedom, however, can potentially supersede its performance.
\end{abstract}

Keywords: Plasmonics; single fluorescence molecule; evanescent field; zero-mode waveguide

Citation: Muhammad Shemyal NISAR, Yujun CUI, Kaitong DANG, Liyong JIANG, and Xiangwei ZHAO, "Near-Field Spot for Localized Light-Excitation of a Single Fluorescent Molecule," Photonic Sensors, 2020, 10(4): 364-374.

\section{Introduction}

Single molecule fluorescence (SMF) is a key technology for many applications in molecular biology, molecular kinetics [1-3], transient intermediates for chemical or biochemical reactions [4, 5], deoxyribonucleic acid (DNA) sequencing [6, 7], diagnostics [8, 9], and personalized medicine $[10,11]$. Generally, there are two means to improve the signal to noise ratio (SNR) by using SMF. One way is to reduce the intrinsic background fluorescence (typically) via chemical means. The other method is to reduce the excitation volume such that less background noise is generated by physical methods. For example, laser scanning confocal microscopy (LSCM) [12, 13], total internal reflection microscopy (TIRF) $[14,15]$, waveguide evanescent field fluorescence microscopy [16, 17], two-photon microscopy [18], and stimulated emission depletion (STED) microscopy [19] use optical settings to confine the excitation to a very small volume. There are also other techniques that use nanostructures for the localized excitation of fluorescence, for example, Raman-signal-like scanning near-field optical microscopy (SNOM) [20], tip enhanced Raman scattering (TERS) [21], optical 
nanoantennas/nanocavities $[22, \quad 23], \quad$ and zero-mode waveguides (ZMWs) [24-26].

By reducing the excitation volume to $10^{-21}$ liter at the bottom of a metal nanohole, ZMW observes single fluorescent molecules, at biologically relevant concentrations as well as up to the millimolar range. The ZMW could also enable fluorescence correlation spectroscopy (FCS) [25-27]. Therefore, ZMWs and their variations have been used in various applications including the analysis of single molecules at high concentrations [27], single cells [28], single enzymes [29], DNA sequencing from polymerase molecules [26, 30], DNA sorting in nano-fluidic channels [31], oligomerization kinetics [2], and redox reactions $[32,33]$.

However, in a ZMW, the nanohole is tens of nanometers in diameter, about $100 \mathrm{~nm}$ in depth, and only the volume of $10 \mathrm{~nm}-20 \mathrm{~nm}$ at the bottom is excited. It is very difficult for biomolecules like enzymes or DNA to drop in the nanohole due to steric effects and a static double layer on the nanohole surface. To overcome this, tilted sidewalls were used, which made the mouth of the ZMW wider than its base, so that the introduction of analyte into the ZMW was facilitated [32, 34]. Alternatively, the potential was applied to different layers of the structure to perform spectro-electrochemical analysis during

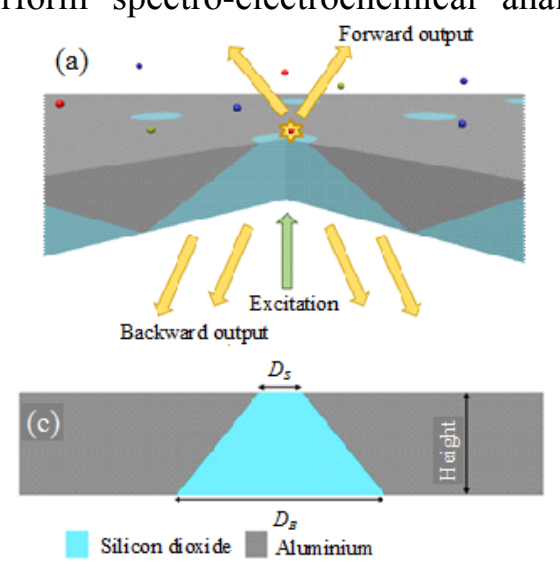

(a) (b) . redox reactions [32]. In another study, a nanopore was embedded at the bottom of the nanohole, and a voltage was applied to introduce DNA strands into the ZMW and capture them via DNA polymerase for sequencing [30]. However, the complexity and cost of the ZMW increased. This means that it is still challenging to excite and collect the fluorescence signal of a single molecule in a zeptoliter volume with high accessibility.

In this paper, we investigate and numerically simulate a novel plasmonic structure, the near-field spot (NFS), which confines the excitation of a single fluorescence molecule into a zeptoliter volume on an open planar surface. As illustrated in Fig. 1, the NFS is a nanostructure with tapered metal walls filled with a dielectric material, which forms a planar structure. The apex of the dielectric filling has a width of $50 \mathrm{~nm}$. In this study, we analyze two different morphologies of the NFS, the conical morphology and the pyramidal morphology, and we compare them with ZMWs [Fig. 1(c)]. Since the surface chemistry of silica is different from a metallic component, it is favorable for the selective immobilization of biomolecules in the NFS, when it is used for FCS or DNA sequencing. Moreover, two possible schemes for a practical experimental analysis are provided in Figs. 2 and 3.
Forward output
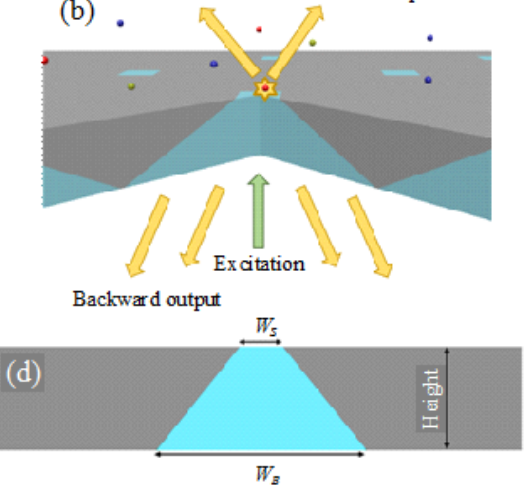

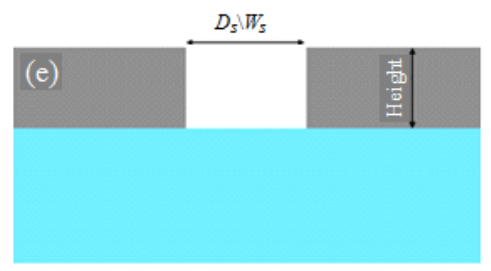

Fig. 1 Operational layout of the device and architectural schematic diagrams: (a) schematic diagram of the NFS using conical morphology (side view), (b) pyramidal morphology along with the molecules, (c) 2-dimensional (2D) schematic diagram of the NFS showing various dimensions of the structure for the conical morphology, (d) pyramidal morphology, and (e) 2D schematic diagram showing a comparable ZMW with a height equivalent for the corresponding NFS, and a width equivalent to $D_{s}$ for a conical morphology and $W_{s}$ for a pyramidal morphology. 


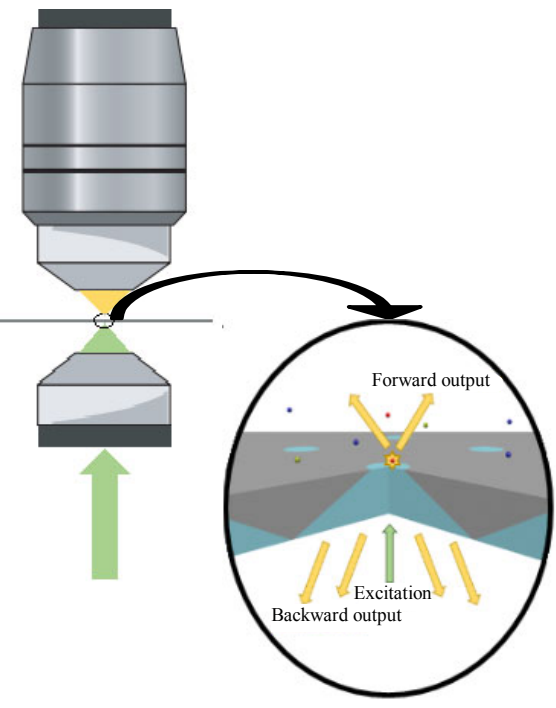

Fig. 2 Scheme for experimental setup for analysis using NFS (this scheme uses forward scattered fluorescent signal for observation)

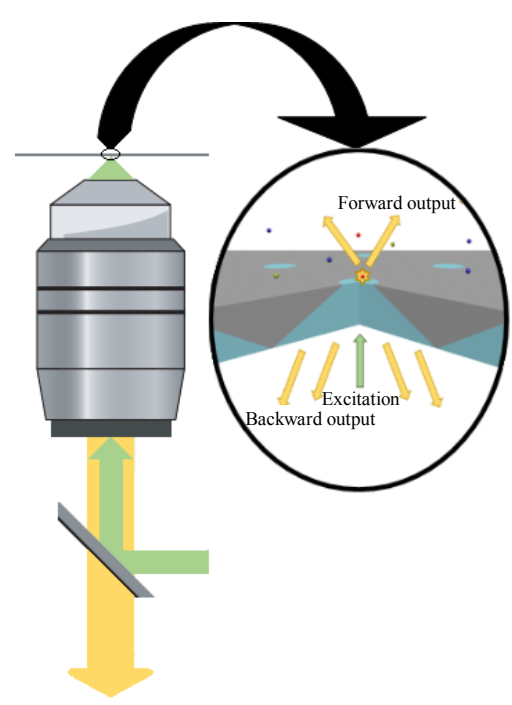

Fig. 3 Scheme for experimental setup for analysis using NFS (this scheme uses backward scattered fluorescent signal for observation).

The focusing of the incident field can be understood through the metal/dielectric boundary conditions and the dynamics of plasmonic modes (surface plasmon polaritons - SPP) at the interface between the metal and the dielectric. In a metalinsulator-metal system (MIM), SPPs propagation obeys the well-known set of equations [21,35] with a cut-off wavelength [35]. All of these equations are presented in (1) below. The results from this work conform well with other literatures on plasmonic nanofocusing by authors such as Eli Yablonovick [36] and others [21,37].

$$
\begin{gathered}
k_{\mathrm{spp}}=\frac{2 \pi}{\lambda_{o}} \sqrt{\frac{\varepsilon_{m} \varepsilon_{d}}{\varepsilon_{m}+\varepsilon_{d}}} \\
\lambda_{p}=\frac{2 \pi}{\operatorname{Re}\left[k_{\mathrm{spp}}\right]} .
\end{gathered}
$$

where $\epsilon_{m}$ and $\epsilon_{d}$ are the dielectric constants for the metal and dielectric, respectively, $k_{\mathrm{spp}}$ is the wave vector, $\lambda_{o}$ is the wavelength of the incident light, and $\lambda_{p}$ is the wavelength of the plasma. It is clear from these equations that the wave vector of the resulting plasmons is inversely related to the incident wavelength. The relationship is further mediated by the permittivity of the metal as well as the permittivity of the dielectric material. Equation(1b) highlights the relationship between the wavelength of the surface plasmon and its wave vector.

The NFS is deemed advantageous compared with a ZMW because it is a planar structure, which removes the need for additional mechanisms to introduce the desired molecule into the nanopore of the ZMW without compromising device performance. Moreover, the architecture of a ZMW provides more degrees of freedom to improve the performance and can theoretically achieve a higher electric field intensity than a ZMW.

\section{Numerical simulation}

Finite difference time domain (FDTD) is used for the numerical simulation of the NFS. The top surface of the NFS has water with a refractive index of 1.33 , while the bottom surface is in air with a refractive index of 1 . The refractive index of silicon dioxide is chosen to be 1.45 , with optical constants for aluminum taken from Palik. To find a balance between accurate results and the resources required by the system for the simulation, a manually adjusted non-uniform mesh is used with two levels of description. For the region around the top surface, 
a cubic mesh element, with a uniform size of $2 \mathrm{~nm}$, is used to improve the accuracy of the results. For the rest of the structure, an automatic non-uniform mesh is used. To simplify the simulation, the simulation region is increased in lateral directions, and perfectly matched layer (PML) boundary conditions are applied to all boundaries. To make the simulation less expensive with respect to computational resources, a single wavelength $x$-polarized Bloch/periodic plane-wave with the wavelength of $532 \mathrm{~nm}$ and amplitude of $1 \mathrm{~V} \cdot \mathrm{m}^{-1}$ is used as the excitation source. The choice of the wavelength of the excitation source is arbitrary, and changing it does not affect the general principles we explain here. To obtain useful results from this simulation setup, two 2D field and power monitors are used. One shows the electric field in the whole structure, while the other shows the region above the NFS. A separate monitor above the NFS is used to directly observe the evanescent field produced by the structure. This would otherwise be difficult to observe in the full-sized image.

Similarly, for comparison, a simulation model of a conventional ZMW is set up, using silicon dioxide and aluminum in water [Fig. 1(e)]. The width of the top of the NFS and the width of the ZMW are chosen to be identical. Moreover, the thickness of this ZMW is chosen to be equivalent to the NFS to make both of these models comparable.

Finally, the ability of the structure to collect the fluorescence is an important design consideration. This is explored by setting up a point-dipole source in the region of analysis, right above the NFS. The electric field, which is emitted from the dipole, is then collected from the base of the structure to determine the proportion of the electric field that can be received by the observer.

\section{Results and discussion}

\subsection{Conical morphology}

For the conical morphology, the diameter at the top surface, $D_{s}$, is $50 \mathrm{~nm}$, while the diameter at the bottom, $D_{B}$, changes from $50 \mathrm{~nm}$ to $500 \mathrm{~nm}$ to enable different degrees of nano-focusing of light. Both the thickness of metal and the height of the section of the cone are kept equal. For the current analysis, two different heights, $50 \mathrm{~nm}$ and $100 \mathrm{~nm}$, are used for the cone. The schematic diagrams of structures of the NFS for the conical and pyramidal morphologies and a comparable ZMW are shown in Fig. 1.

The first step is to rule out any possibility of leakage of the electric field through the metal film as the distance between the dielectric/metal interface and the metal/water interface falls below the metal's skin depth in a structure with large $D_{s}$. The skin depth of aluminum at $532 \mathrm{~nm}$ is approximately $3.5 \mathrm{~nm}$. Because the region with a metal thickness below $3.5 \mathrm{~nm}$ is located very close to the apex, it can be safely assumed that there is no significant leakage occurring through the metal. To confirm this, the structure is set up in such a way that $z=0$ overlaps with the water/dielectric interface for the NFS as well as for the ZMW. The numerical simulation is carried out, and the electric field profile conforms with the shape of the structure, as shown in Fig. 4(a). For this simulation, $D_{B}=500 \mathrm{~nm}$ is chosen because the gradient of its silicon dioxide/aluminum interface is the smallest among the samples. The results indicate that there is no significant leakage of the electric field from the structure, and the profile of the NFS allows the incoming light to focus on the nanometer-scale aperture.

After satisfying these initial concerns, we study the dependence of the electric field intensity on the distance along the $z$-axis for all structures. The dependence of the electric field intensity on the distance along the $z$-axis is shown in Fig. 5. These results reveal two important features. Firstly, the electric field intensity of the structure increases with increasing $D_{B}$. This increase also increases the range of the electric field because for the NFS (with $D_{B}$ of 
$100 \mathrm{~nm}$ and above) the electric field is higher than that of the ZMW for all distances. Secondly, because the electric field in a ZMW is rapidly quenched by its metal walls [27, 37], its intensity decreases very rapidly compared with the NFS, which does not have the metal wall. As a result, even the electric field intensity for the NFS with $D_{B}=50 \mathrm{~nm}$ at the interface is below ZMW. Above the $25 \mathrm{~nm}$ mark (approx.), however, the electric field of the ZMW decreases to levels below that of the NFS.
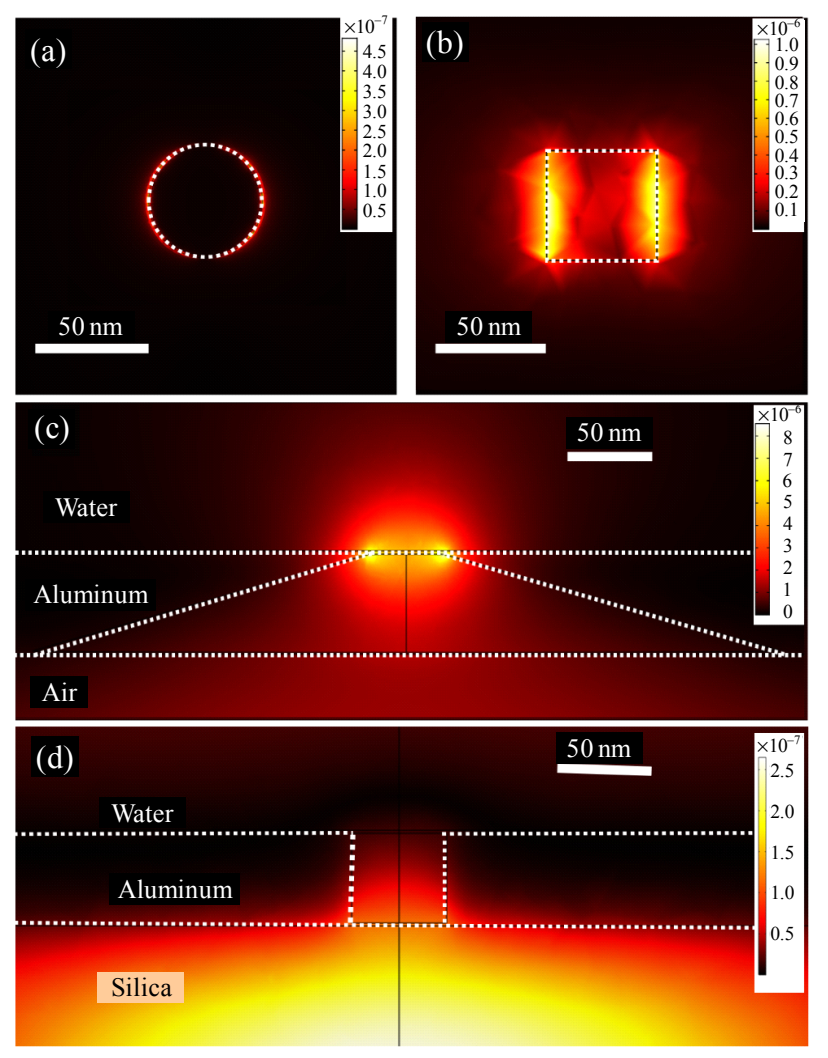

Fig. 4 Intensity profile of the electric field at $z=0$ : (a) conical morphology and (b) pyramidal morphology; (c) electric field profile of a near-field spot with the width of $50 \mathrm{~nm}$ at the apex and a height of $100 \mathrm{~nm}$; (d) electricfield profile of a ZMW with a width of $50 \mathrm{~nm}$ and a height of $100 \mathrm{~nm}$.

Similar results are obtained from the NFS with a height of $100 \mathrm{~nm}$. Figure 5(b) shows the electric field intensity from all structures along the $z$-axis, when the height of the structures (NFS as well as ZMW) increases to $100 \mathrm{~nm}$. However, the effect on the performance of the NFS is disproportionately stronger than that of a ZMW. The reduction of the electric field intensity with an increase in height may be attributed to increased attenuation due to prolonged interaction with the aluminum sidewalls. The peak intensity of the ZMW suffers disproportionately less from an increase in height compared with the NFS because of two reasons. Firstly, a ZMW confines the light to a very small space, which is defined by metals walls, while there is no such confinement for the NFS. The metal walls, which cause the rapid attenuation of the electric field in a ZMW, improve its intensity at the interface. For these reasons, the electric field intensity from the ZMW stays almost the same at the interface, while the intensity from the NFS is heavily attenuated. Secondly, for a ZMW, the incoming electric field does not interact with the metal walls until it reaches the cavity. This is also the same place as the observation area for a ZMW. On the other hand, for the NFS, the cavity with a metal wall is used for confinement and focusing the electric field, while the observation area is above this cavity. Therefore, at the interface, the intensity of even the NFS of $D_{B}=100 \mathrm{~nm}$ is below that of the ZMW. However, as for the earlier case, this advantage of the ZMW is quenched rapidly.

\subsection{Pyramidal morphology}

Similar to the conical morphology, for the pyramidal morphology, the width of the apex, $W_{S}$, is $50 \mathrm{~nm}$, while the bottom width, $W_{B}$, varies between $50 \mathrm{~nm}$ and $500 \mathrm{~nm}$. The thickness of the metal and the height of the pyramid are kept equal. For the current analysis, two different pyramid heights, $50 \mathrm{~nm}$ and $100 \mathrm{~nm}$, are used. The schematic diagrams of the structures of the NFS with pyramidal morphology and a comparable ZMW are shown in Fig. 1. The simulation is set up as for the conical structure. $Z=0$, therefore, overlaps with the water/dielectric interface of the NFS as well as the ZMW. The dependence of the electric field intensity on the distance along the $z$-axis is recorded for all structures. 


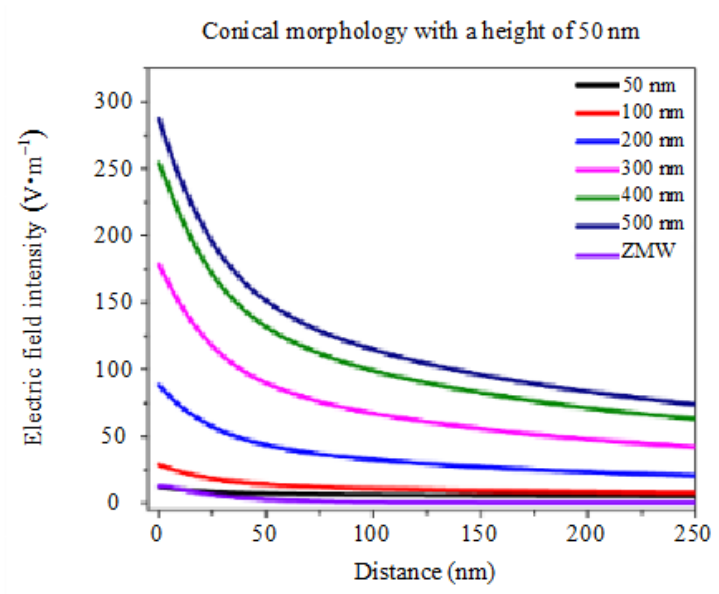

(a)

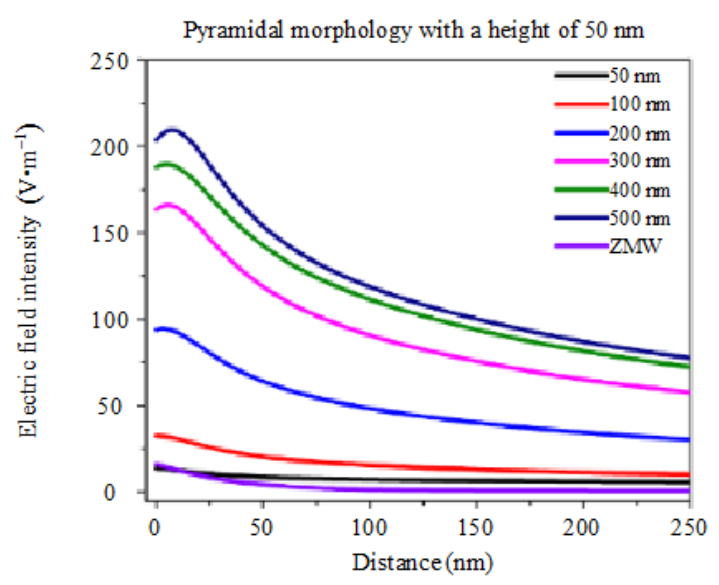

(c)

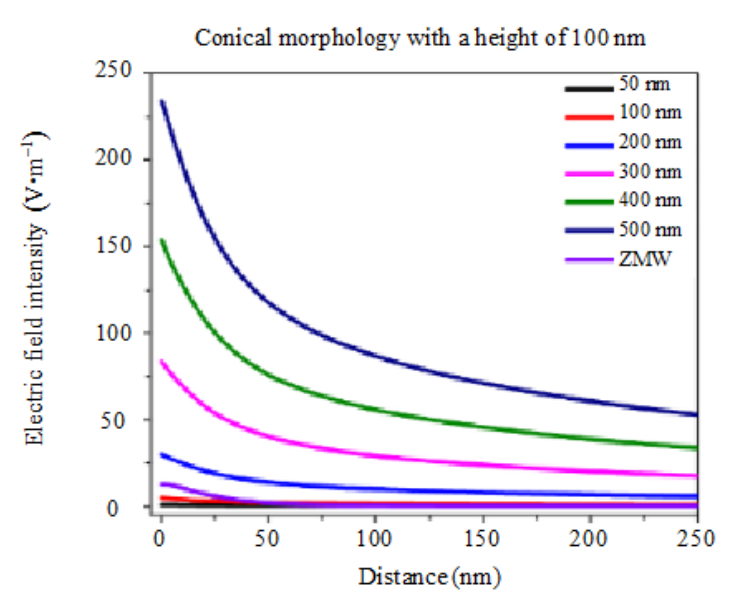

(b)

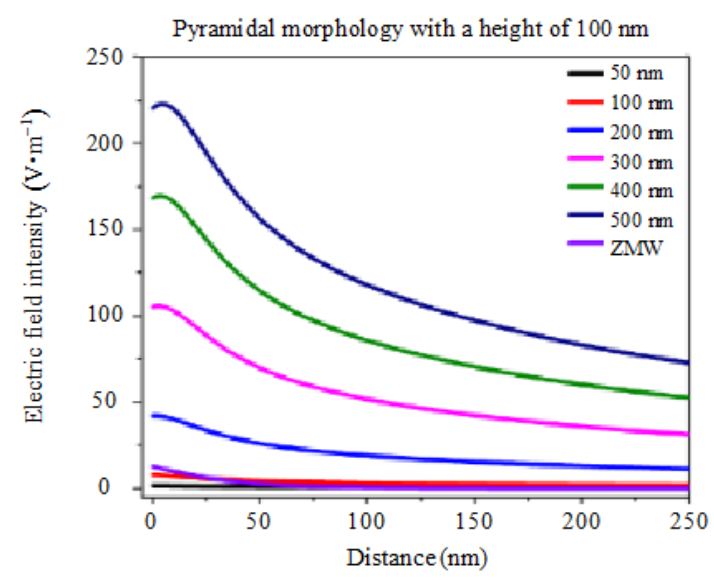

(d)

Fig. 5 Comparison of the near field electric field intensity along the $z$-axis distance from the water/dielectric interface of: (a) the conical NFS with a different $D_{B}$ and a ZMW with a diameter of $50 \mathrm{~nm}$ and a height of $50 \mathrm{~nm}$, (b) the conical NFS with a different $D_{B}$ and a ZMW with a diameter of $50 \mathrm{~nm}$ and a height of $100 \mathrm{~nm}$, (c) the pyramidal NFS with a different $W_{B}$ and a ZMW with a diameter of $50 \mathrm{~nm}$ and a height of $50 \mathrm{~nm}$, and (d) pyramidal NFS with a different $W_{B}$ and a ZMW with a diameter of $50 \mathrm{~nm}$ and height of $100 \mathrm{~nm}$.

As for the conical NFS, the first test is to confirm that there is no leakage from the metal film, as shown in Fig. 4(b). The profile of the electric field at the interface agrees with the shape and size of the cavity sufficiently well.

Next, the performance of the pyramidal NFS is compared with a square ZMW. The dependence of the electric field intensity on the distance from the interface is shown in Fig. 5(c). For the pyramidal structure, the results are similar to those of the conical structure. Figure 5(c) shows that for the pyramidal NFS, with a height of $50 \mathrm{~nm}$, the electric field intensity decreases with the distance along the $z$-axis. However, there is an increase in electric field intensity with increasing $W_{B}$. Compared with a square ZMW, the evanescent field of the NFS with $W_{B}=50 \mathrm{~nm}$ is smaller. The ZMW intensity is quenched rapidly, and around the $40 \mathrm{~nm}$ mark, the electric field of the NFS becomes stronger than that of the ZMW. Moreover, when $W_{B}$ of the NFS increases to $100 \mathrm{~nm}$, its performance is comparable with the ZMW at the interface and better than the ZMW beyond it.

By increasing the height of the NFS and the 
ZMW to $100 \mathrm{~nm}$, we observe [Fig. 5(d)] that the electric field intensity of a ZMW again stays approximately the same, while that of the NFS decreases. The reasons for this have been explained earlier.

\subsection{Collecting fluorescence from the NFS}

For the NFS to have any practical value, the collection of fluorescence is important. Unlike the ZMW, the NFS supports two different methods of fluorescence collection, as shown in Fig. 6(i). The first method is "forward output", which is the method of looking at the fluorescence directly from the top. Given that two consecutive spots are about at least a micron away from each other, capturing and distinguishing the response from the individual spot is practically possible. A possible experimental scheme for this method is shown in Fig. 2 .

The second method is "backward output", as shown in Fig. 6(i). This method is the same as the one used for the ZMW. The generated fluorescent light travels through the structure and is

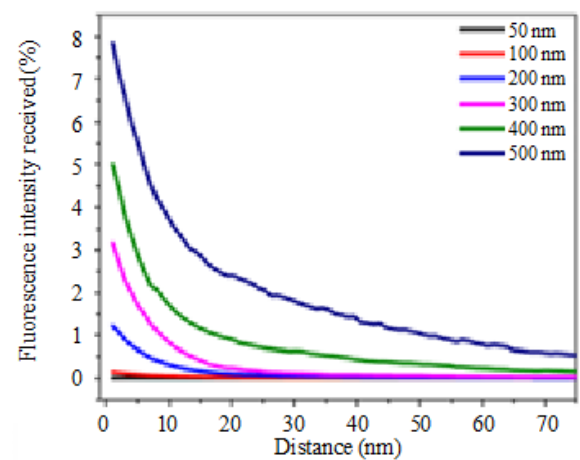

(a)

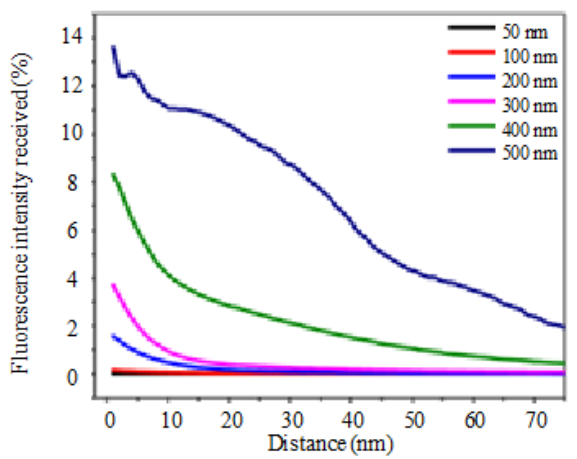

(c) subsequently collected at the bottom by using well-known methods [27, 31]. For our analysis, we use the backward output for two systems to be equivalent. We set up the system with a dipole at its apex, which represents a fluorescently tagged biomolecule on the top of the NFS. The fluorescent light emitted from the dipole travels through the structure and is collected at the bottom of the device. Many fluorescent tags are known that they emit fluorescence signals with different frequencies. For this study, considering the application of DNA sequencing, two different wavelengths, $532 \mathrm{~nm}$ and $637 \mathrm{~nm}$, and a dipole moment of $6.2 \times 10^{-30} \mathrm{~cm}$ are used. This dipole is moved along the $z$-axis to gauge the dependence of the electric field collected at the distance of the dipole from the NFS. Similarly, the $D_{B} / W_{B}$ changes as well to measure the dependence of the electric field collected within the diameter of the base of a conical NFS and the width of the base of a pyramidal NFS. A possible experimental scheme for this method is shown in Fig. 3.

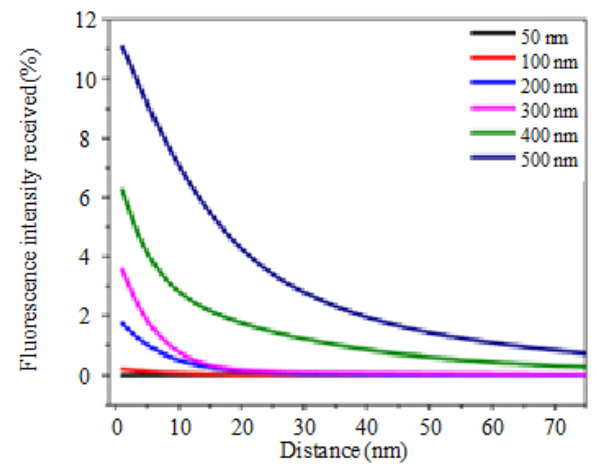

(b)

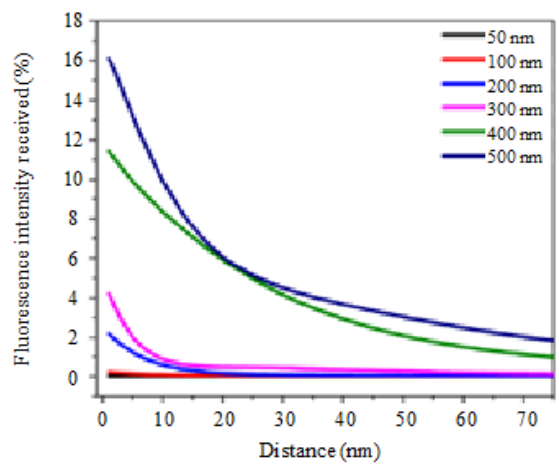

(d) 


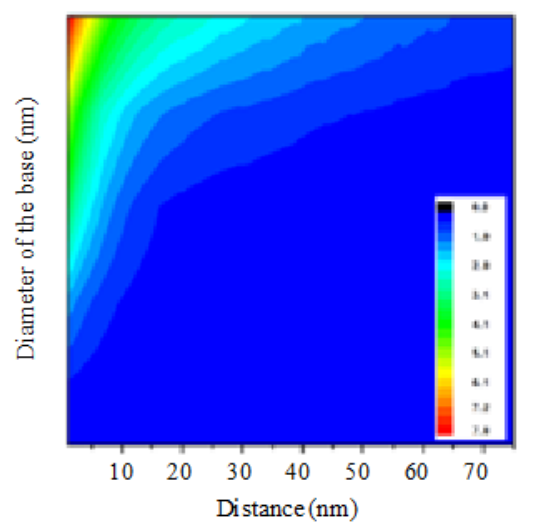

(e)

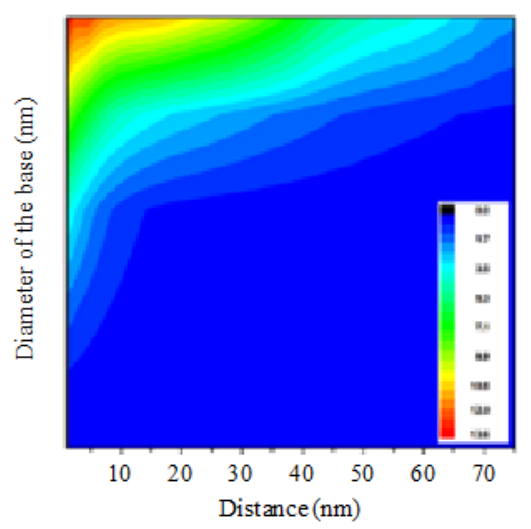

(g)

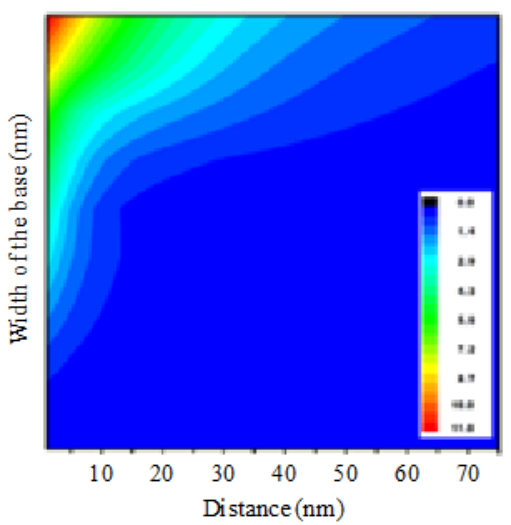

(f)

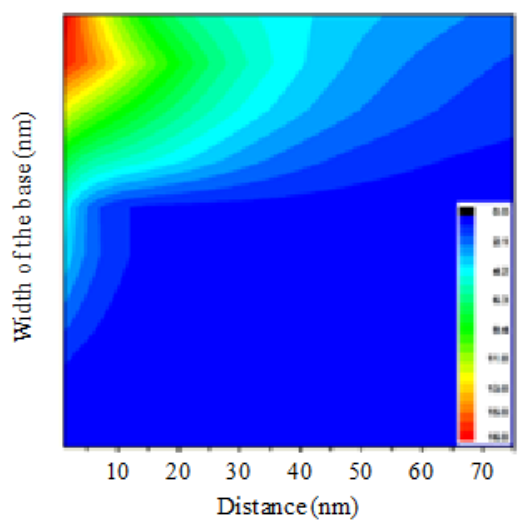

(h)

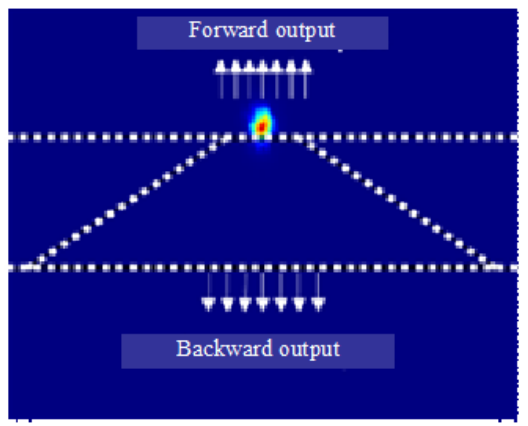

(i)

Fig. 6 Electric field intensity received at the base of the NFS as a percentage of power transmitted from a dipole from the NFS with different $D_{B} / W_{B}$ : (a) with conical morphology $(\lambda=637 \mathrm{~nm})$, (b) with pyramidal morphology $(\lambda=637 \mathrm{~nm}),($ c) with conical morphology $(\lambda=532 \mathrm{~nm})$, (d) with pyramidal morphology $(\lambda=532 \mathrm{~nm})$; contour maps showing the relationship of electric field intensity, received at the base of the NFS, with a dipole distance from the NFS and $D_{B} / W_{B}$ of the NFS: (e) with conical morphology $(\lambda=637 \mathrm{~nm})$, (f) with pyramidal morphology $(\lambda=637 \mathrm{~nm}),(\mathrm{g})$ with conical morphology $(\lambda=532 \mathrm{~nm})$, (h) with pyramidal morphology $(\lambda=532 \mathrm{~nm})$; (i) electric field profile of the dipole showing the simulation setup and the mechanism of receiving the spectrum.

The electric field, which is received from a dipole of $532 \mathrm{~nm}$ in conical structures as well as pyramidal structures, is shown in Figs. 6(c) and 6(d).
The performance of the pyramidal structural morphology is better than that of the conical structural morphology. This is because the 
maximum received electric field intensity (in the case of the pyramidal structure) exceeds $16 \%$ of the initial electric field intensity transmitted from the dipole, whereas the received electric field intensity (using a conical structure) is around 14\% of the initial electric field intensity emitted from the dipole. Moreover, the electric field intensity received at the base of each structure decreases with the increasing diameter/width of the base of the NFS. This is because, as the diameter/width of the base increases, the rate of change in the diameter/width to distance (along the central axis of the cone/pyramid) increases. An increase in the rate of change of the diameter helps increase the propagation length of the electric field and decrease losses as the electric field propagates along the metal/dielectric interface as a surface plasmon polariton.

Similarly, for the fluorescence signal with a wavelength of $637 \mathrm{~nm}$, the performances of both (conical and pyramidal) structures are numerically calculated [see Figs. 6(a), 6(b), 6(e), and 6(f)]. Overall, the performance of both structures worsens for the dipole with a wavelength of $637 \mathrm{~nm}$. This is because the propagation length decreases with increasing wavelength, and therefore, propagation losses increase with increasing wavelength. As for the $532 \mathrm{~nm}$ wavelength, the performance of the pyramidal NFS is better than that of the conical NFS for each diameter/width of the NFS. The electric field intensity from the dipole, received at the base of these structures, increases with increasing diameter/width of the NFS. The maximum electric field intensity, which is received at the base of the pyramidal NFS, is around $11 \%$. However, the electric field intensity, which is received at the base of a conical NFS, is around $8 \%$ of the electric field intensity emitted from the dipole.

\section{Conclusions}

We have proposed a planar plasmonic-based platform that can focus light at a sub-wavelength spot, which is called "near-field spot". Furthermore, we demonstrate through numerical simulations that the new platform is comparable with conventional ZMWs. It can even surpass its counterpart, ZMWs, for certain ratios of height and $W_{S}\left(D_{S}\right)$. Given that the NFS is a planar device, it removes the need to implement complex techniques used in the ZMW to help the analyte enter the nano-cavity. The NFS, therefore, has clear advantages over the ZMW. The NFS also allows the designer to tailor the electric field intensity at the apex more effectively, depending on their particular needs. The results also indicate that the improved freedom that the NFS generates can also produce higher electric field intensities at the apex than that of the traditional ZMW. Moreover, the electric field intensity at the interface is better for the pyramidal structure than that for the conical structure.

Particularly, for FCS-based DNA-sequencing applications, the ability of the NFS to transmit the fluorescent signal of multiple frequencies back to the observation unit at the back of the NFS is analyzed. This analysis shows that the pyramidal structure has a slight advantage in transmitting the fluorescent light compared with the conical structure and the performance degrades with increasing wavelength. Furthermore, the transmission of the electric field intensity from the fluorescence source is improved with increasing $W_{B}$ and $D_{B}$ of the particular NFS. The fluorescent collection is slightly less effective than that for a ZMW. Given the advantages of NFS over a ZMW, which come without significant degradation in overall performance, we anticipate that the NFS will be widely used for FCS-based single-molecule analysis and DNA-sequencing applications in the future.

Finally, despite the advantages, the proposed NFS's challenges lie in its physical realization for practical purposes. As it is evident from its intricate 3-dimension (3D) nanoscale design, the fabrication of such a device represents an engineering challenge. Given the currently available state-of-the-art fabrication technologies, such as focused ion beam 
(FIB) and plasma enhanced chemical vapor deposition (PECVD), it appears possible to realize an NFS. The physical realization of the NFS substantially advances the field of single molecule fluorescence.

\section{Acknowledgment}

We acknowledge the support of Southeast University for carrying out this research. This work was financially supported by Southeast University Postdoctoral Science Foundation Grant program, China (Grant No. 2242018R20013), the National Key Research and Development Program of China (Grant No. 2017YFA0205700), the National Natural Science Foundation of China (Grant No. 61850410528), the Fundamental Research Funds for the Central Universities, and the Fundamental Research Project of Shenzhen Science \& Technology Innovation Committee (Grant No. JCYJ20180306174552939).

Open Access This article is distributed under the terms of the Creative Commons Attribution 4.0 International License (http://creativecommons.org/licenses/by/4.0/), which permits unrestricted use, distribution, and reproduction in any medium, provided you give appropriate credit to the original author(s) and the source, provide a link to the Creative Commons license, and indicate if changes were made.

\section{References}

[1] T. Miyake, T. Tanii, H. Sonobe, R. Akahori, N. Shimamoto, T. Ueno, et al., "Real-time imaging of single-molecule fluorescence with a zero-mode waveguide for the analysis of protein-protein interaction," Analytical Chemistry, 2008, 80(15): 6018-6022.

[2] K. T. Samiee, M. Foquet, L. Guo, E. C. Cox, and H. G. Craighead, " $\lambda$-repressor oligomerization kinetics at high concentrations using fluorescence correlation spectroscopy in zero-mode waveguides," Biophysical Journal, 2005, 88(3): 2145-2153.

[3] A. A. Al Balushi and R. Gordon, "A label-free untethered approach to single-molecule protein binding kinetics," Nano Letters, 2014, 14(10): 5787-5791.

[4] W. K. Ridgeway, D. P. Millar, and J. R. Williamson, "Quantitation of ten 30s ribosomal assembly intermediates using fluorescence triple correlation spectroscopy," Proceedings of the National Academy of Sciences, 2012, 109(34): 13614-13619.

[5] E. Kim, M. D. Baaske, I. Schuldes, P. S. Wilsch, and F. Vollmer, "Label-free optical detection of single enzyme-reactant reactions and associated conformational changes," Science Advances, 2017, 3(3): e1603044.

[6] S. Picelli, O. R. Faridani, A. K. Bjorklund, G. Winberg, S. Sagasser, and R. Sandberg, "Full-length RNA-seq from single cells using sart-seq2," Nature Protocols, 2014, 9(1): 171-181.

[7] C. Gawad, W. Koh, and S. R. Quake, "Single-cell genome sequencing: current state of the science," Nature Reviews Genetics, 2016, 17(3): 175-188.

[8] M. A. Walling, J. A. Novak, and J. R. E. Shepard, "Quantum dots for live cell and in vivo imaging," International Journal of Molecular Sciences, 2009, 10(2): 441-491.

[9] S. L. Diedenhofen, D. Kufer, T. Lasanta, and G. Konstantatos, "Integrated colloidal quantum dot photodetectors with color-tunable plasmonic nanofocusing lenses," Light: Science and Applications, 2015, 4(1): e234.

[10] M. Zampieri, K. Sekar, N. Zamboni, and U. Sauer, "Frontiers of high-throughput metabolomics," Current Opinion in Chemical Biology, 2017, 36: 15-23.

[11] C. Dincer, R. Bruch, A. Kling, P. S. Dittrich, and G. A. Urban, Multiplexed point-of-care testing-XPOCT," Trends in Biotechnology, 2017, 35(8): 728-742.

[12] Y. Kozawa, D. Matsunaga, and S. Sato, "Superresolution imaging via superoscillation focusing of a radially polarized beam," Optica, 2018, 5(2): 86-92.

[13] H. Hasegawa, "Laser scanning confocal microscopy," Kobunshi, 2006, 55(12): 961-965.

[14] K. N. Fish, "Total internal reflection fluorescence (TIRF) microscopy," Current Protocols in Cytometry, 2009, 50(1): 12.18.1-12.18.13.

[15] A. Trache and G. A. Meininger, Total internal reflection fluorescence (TIRF) microscopy: current protocols in microbiology, Chapter 2. Hoboken, NJ, USA: John Wiley \& Sons, Inc., 2008.

[16] H. M. Grandin, B. Städler, M. Textor, and J. Vörös, "Waveguide excitation fluorescence microscopy: a new tool for sensing and imaging the biointerface," Biosensors and Bioelectronics, 2006, 21(8): 1476-1482.

[17] A. Hassanzadeh and D. Azami, "Waveguide evanescent field fluorescence microscopy: theoretical investigation of optical pressure on a cell," Journal of Nanophotonics, 2014, 8(1): 083076.

[18] P. T. So, C. Y. Dong, B. R. Masters, and K. M. Berland, "Two-photon excitation fluorescence microscopy," Annual Review of Biomedical 
Engineering, 2000, 2(1): 399-429.

[19] H. Blom and J. Widengren, "Stimulated emission depletion microscopy," Chemical Reviews, 2017, 117(11): 7377-7427.

[20] U. Dürig, D. W. Pohl, and F. Rohner, "Near-field optical-scanning microscopy," Journal of Applied Physics, 1986, 59(10): 3318-3327.

[21] N. C. Lindquist, J. Jose, S. Cherukulappurath, X. Chen, T. W. Johnson, and S. H. Oh, "Tip-based plasmonics: squeezing light with metallic nanoprobes," Laser and Photonics Reviews, 2013, 7(4): 453-477.

[22] J. T. Hugall, A. Singh, and N. F. Van Hulst, "Plasmonic cavity coupling," ACS Photonics, 2018, 5(1): 43-53.

[23] A. Gopinath, E. Miyazono, A. Faraon, and P. W. K. Rothemund, "Engineering and mapping nanocavity emission via precision placement of DNA origami," Nature, 2016, 535(7612): 401-405.

[24] J. M. Moran-Mirabal and H. G. Craighead, "Zero-mode waveguides: sub-wavelength nanostructures for single molecule studies at high concentrations," Methods, 2008, 46(1): 11-17.

[25] T. Plénat, S. Yoshizawa, and D. Fourmy, "DNA-guided delivery of single molecules into zero-mode waveguides," ACS Applied Materials and Interfaces, 2017, 9(36): 30561-30566.

[26] T. Auger, J. Mathé, V. Viasnoff, G. Charron, J. M. Di Meglio, L. Auvray, et al., "Zero-mode waveguide detection of flow-driven DNA translocation through nanopores," Physical Review Letters, 2014, 113(2): 028302 .

[27] M. J. Levene, J. Korlach, S. W. Turner, M. Foquet, H. G. Craighead, and W. W. Webb, "Zero-mode waveguides for single-molecule analysis at high concentrations," Science, 2003, 299(5607): 682-686.

[28]C. I. Richards, K. Luong, R. Srinivasan, S. W. Turner, D. A. Dougherty, J. Korlach, et al., "Live-cell imaging of single receptor composition using zero-mode waveguide nanostructures," Nano Letters, 2012, 12(7): 3690-3694.
[29] Y. Morita, K. Fujimoto, R. Iino, M. Tomishige, H. Shintaku, H. Kotera, et al., "Single-molecule fluorescence imaging of kinesin using linear zero-mode waveguides," in 2016 IEEE Sensors, USA, Oct. 30 - Nov. 3, 2016, pp: 1-3.

[30] J. Larkin, R. Y. Henley, V. Jadhav, J. Korlach, and M. Wanunu, "Length-independent DNA packing into nanopore zero-mode waveguides for low-input DNA sequencing," Nature Nanotechnology, 2017, 12(12): $1169-1175$.

[31] J. Eid, A. Fehr, J. Gray, K. Luong, J. Lyle, G. Otto, et al., "Real-time DNA sequencing from single polymerase molecules," Science, 2009, 323(5910): 133-138.

[32] D. Han, G. M. Crouch, K. Fu, L. P. Zaino, and P. W. Bohn, "Single-molecule spectroelectrochemical cross-correlation during redox cycling in recessed dual ring electrode zero-mode waveguides," Chemical Science, 2017, 8(8): 5345-5355.

[33] G. M. Crouch, D. Han, and P. W. Bohn, "Zero-mode waveguide nanophotonic structures for single molecule characterization," Journal of Physics D: Applied Physics, 2018, 51(19): 193001.

[34] L. P. Zaino, D. A. Grismer, D. Han, G. M. Crouch, and P. W. Bohn, "Single occupancy spectroelectrochemistry of freely diffusing flavin mononucleotide in zero-dimensional nanophotonic structures," Faraday Discussions, 2015, 184(1): $101-115$.

[35] R. Gordon and A. G. Brolo, "Increased cut-off wavelength for a subwavelength hole in a real metal," Optics Express, 2005, 13(6): 1933-1938.

[36] H. Choo, M. K. Kim, M. Staffaroni, T. J. Seok, J. Bokor, S. Cabrini, et al., "Nanofocusing in a metal-insulator-metal gap plasmon waveguide with a three-dimensional linear taper," Nature Photonics, 2012, 6(12): 838-844.

[37] R. Gordon, A. G. Brolo, D. Sinton, and K. L. Kavanagh, "Resonant optical transmission through hole-arrays in metal films: physics and applications," Laser and Photonics Reviews, 2010, 4(2): 311-335. 